\title{
Primary pulmonary alveolar proteinosis
}

\author{
Gayani GGA' ${ }^{1}$, Karunaratna WGSG ${ }^{1}$, Dilhani PDW ${ }^{1}$, Muhunthan S $^{1}$, Herath HMM${ }^{2}$, Weerarathna TP² \\ ${ }^{1}$ Teaching Hospital Karapitiya, Galle, Sri Lanka \\ ${ }^{2}$ Department of Medicine, Faculty of Medicine, University of Ruhuna, Galle, Sri Lanka
}

Correspondence: Dr.G.G.A.Gayani;

Mobile:+94777120360; e-mail: aysh2002@gmail.com

\begin{abstract}
Introduction
Pulmonary alveolar proteinosis, a rare pulmonary disorder, was first described by Rosen et al in 1958 (1). It is characterised by abnormal accumulation of surfactant derived substances in the alveoli, due to a defect either in the production of surfactant or the clearance by alveolar macrophages, which explains the high association with malignancies and unusual infections. Those affected could have mild symptoms, spontaneous resolution or progressive disease with respiratory failure.
\end{abstract}

\section{Case report}

A 35-year-old female presented with progressively worsening exertional dyspnoea for six months. She also complained of chronic cough, low grade fever for two months, pleuritic-type chest pain, loss of appetite, fatigue, malaise and a marked weight loss (27 kg over 6 months). She gave a history of exposure to dust at her domestic employment of manufacturing bricks. She had never smoked but exposed to passive smoking at home. Screening for tuberculosis in the previous month had been negative. Despite the negative tests, she had been treated with specific anti-TB drugs owing to the nature of the symptoms and other findings.

On physical examination, she was afebrile, tachypnoeic, centrally cyanosed with a $\mathrm{SaO}_{2}$ of $65 \%$ on air. There were few scattered crepitations bilaterally.

Arterial blood gas analysis revealed Type I respiratory failure with $\mathrm{PCO}_{2} 29 \mathrm{mmHg}$ and $\mathrm{PO}_{2}$ $63 \mathrm{mmHg}$. Lung function tests showed restrictive lung disease (Figure 1).

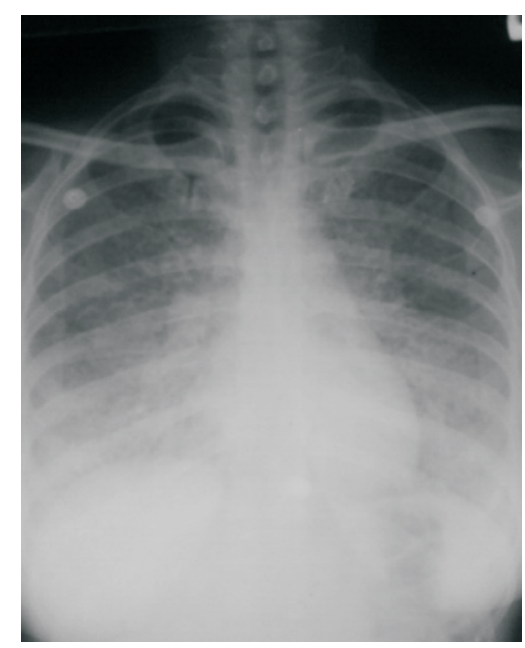

Figure 1: Chest radiograph showing bilateral interstitial and alveolar shadows

Sputum for gram stain and culture didn't show any pathogens and three sputum samples were negative for acid fast bacilli. There were no malignant cells in sputum cytology. Her lactate dehydrogenase was 397 U/L ( 85 - 234) and HIV I and II antibodies were negative. Other laboratory tests revealed normal haematological parameters with normal kidney and liver functions (Figure 2).

Her bronchoalveolar lavage fluid analysis showed numerous, round to irregular, hyaline periodic acid Schiff (PAS) positive casts. After evaluating her clinical, radiological and histological information the diagnosis of primary pulmonary alveolar proteinosis was made.

She was managed, initially, with high flow oxygen to maintain the $\mathrm{SaO}_{2} 92 \%$ and subsequently whole lung lavage under anaesthesia. After two cycles of lavage therapy her functional capacity improved and oxygen saturation improved markedly ( $96 \%$ on air). 


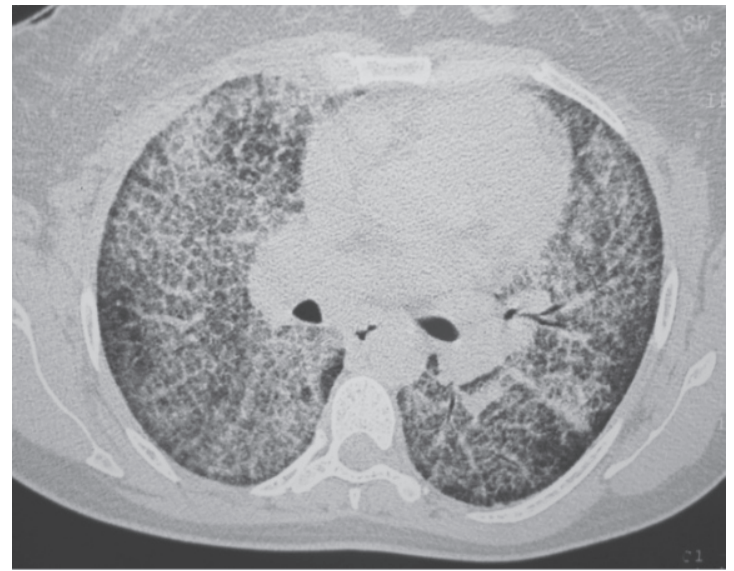

Figure 2: High resolution CT chest showing bilateral extensive ground glass opacities with associated diffuse small smooth intra lobar septal thickening (crazy paving) in both lungs fields

\section{Discussion}

Pulmonary alveolar proteinosis (PAP) is a rare disease with a prevalence of a 0.1 per 100,000 population. It occurs in all age groups and most common in men (male : female $4: 1$ ) and among people aged 20 to 50 years. The disease is commoner among smokers (3 times) than among non-smokers.

There are three clinically distinct forms of PAP. They include congenital $(2 \%)$, primary $(90 \%)$ and secondary $(5-10 \%)$. Congenital PAP is due to a heterogeneous mutation of genes encoding surfactant proteins. (2,3). Primary PAP is considered an autoimmune condition with excess surfactant production caused by GM CSF neutralizing antibodies, receptor deficiency or gene deficiency / mutation, which lead to lack of macrophage stimulation. Immature alveolar macrophages are incapable of proper surfactant clearance. Secondary PAP develops in association with conditions like haematological malignancies, inhalation of toxic dust, fumes or gases, infections or pharmacologic immunosuppression and lysinuric protein intolerance.

PAP can be asymptomatic or it can be more chronic with dyspnoea and cough. Cough is usually dry, but sometimes accompanied by white, tenacious and sticky sputum. Fever and weight loss also can occur. Physical examination is typically nonspecific. Rarely cyanosis, clubbing, crepitations can be seen. Radiological findings are nonspecific with typical finding of bilateral central and symmetrical opacities in interstitial and alveolar spaces. High resolution CT chest revealed bilateral extensive ground glass opacities with associated small smooth intra lobar septal thickening (crazy paving).

Elevated LDH levels are nonspecific in PAP. GMCSF auto antibodies are elevated in primary PAP, but normal in secondary and congenital PAP. Pulmonary function tests reveal restrictive pattern of lung disease, decreased CO diffusion capacity, increased alveolar-arterial partial oxygen pressure gradient, hypoxaemia and elevated shunt fraction.

Open lung biopsy is the gold standard for the diagnosis of PAP. It shows characteristic milky fluid containing large amounts of granular acellular eosinophilic proteinaceous material with foamy macrophages engorged with PAS positive intracellular inclusions.

Treatment of PAP depends on the physiological impairment, rate of progression or remission and the underlying pathology. Whole lung lavage under general anaesthesia is the gold standard treatment for primary PAP. For congenital PAP supportive treatment and occasionally, lung transplantation is practiced, while conservative therapy and management of underlying condition is reserved for secondary PAP (4). In conclusion, the differential diagnosis of PAP should be considered in a patient who comes with chronic cough and dyspnoea in whom initial investigations negative for common causes. A common mistake is to treat them as pulmonary tuberculosis leading to delay in correct diagnosis and specific treatment. Although pulmonary tuberculosis is a common disease, the possibility of PAP should be considered when laboratory tests fail to confirm TB etiology and the expected therapeutic response with anti-TB drugs is not seen during the follow up.

\section{References}

1. Rosen SH, Castleman B, Liebow AA. Pulmonary alveolar proteinosis. NEngl JMed 1958; 258:1123-42.

2. Goldstein LS, Kavuru MS, Curtis-McCarthy P, Christie HA, Farver C, Stoller JK. Pulmonary Alveolar Proteinosis. Clinical features and outcomes. Chest 1998; 114: 1357-62.

3. Trapnell BC, Whitsett JA, Nakata K. Pulmonary Alveolar Proteinosis. NEngl JMed 2003; 349: 2527-9.

4. Huizar I, Kaviru MS. Alveolar proteinosis syndrome. pathogenesis, diagnosis, and management. Opinion in Pulmonary Medicine 2009; 15: 491-8. 